\title{
Malaria among pregnant women in Abeokuta, Nigeria
}

\author{
O.A. IDOWU ${ }^{*}$, C.F. MAFIANA ${ }^{1} \&$ S. DAPO $^{2}$ \\ ${ }^{1}$ Department of Biological Sciences, University of Agriculture, P.M.B. 2240, Abeokuta, Nigeria \\ ${ }^{2}$ Department of Obstetrics and Gynaecology, Federal Medical Centre, Abeokuta, Nigeria
}

\begin{abstract}
Pregnant women were enrolled from two hospitals and a Traditional Birth Home (TBH) in Abeokuta, Nigeria and monitored monthly for malaria parasites from the first antenatal visit until delivery. A total of 466 pregnant women were recruited for the study. The prevalence of malaria was $57.4 \%$, and was higher among women in the TBH. The prevalence of malaria in the first, second and third trimesters of pregnancy were $37.5 \%, 47.3 \%$ and $47.5 \%$ respectively. At the time of the first antenatal visit $35.6 \%$ of the women were already parasitaemic, with a high frequency observed among primigravids. The prevalence recorded in the ninth month of pregnancy $(35.7 \%)$ was not statistically different from that recorded at first antenatal visit $(P>0.05)$. The need to improve the quality of care, malaria prevention and treatment given to pregnant women especially receiving antenatal care outside the hospital is recommended.
\end{abstract}

Keywords: Malaria, pregnancy, antenatal care, traditional birth home, Nigeria

\section{Introduction}

The worldwide annual prevalence of malaria is estimated to be in the order of 300-500 million clinical cases while mortality is estimated to be over 1 million deaths (WHO, 1998). The vast majority of deaths occur among young children in Africa especially in remote rural areas with poor access to health services (UNICEF, 2000). In most endemic areas of the world, pregnant women are the main adult risk group for malaria (Ebrahim, 1996).

Malaria infection during pregnancy has also been a major public health problem in tropical and subtropical regions throughout the world. Every year, at least 30 million women in malarious areas of Africa become pregnant most of which live in areas of relatively stable malaria transmission. The physiological changes of pregnancy and the pathological changes due to malaria have a synergistic effect on each other. The risk of abortion, stillbirth, premature delivery, intrauterine growth retardation and low birth weight has been associated with malaria infection in pregnancy (Leontsini, 1994; Taha et al., 1993; Ebrahim, 1996; Steketee, 1996).

This study was undertaken to determine the prevalence of malaria infection among pregnant women attending antenatal clinics and traditional birth home in Abeokuta, Nigeria.

\section{Materials and Methods}

The study was carried out in Abeokuta, Ogun State, Nigeria and involved pregnant women attending antenatal clinics at Federal Medical Centre, Ogun State General Hospital and a Traditional Birth Home (in order to include women not receiving antenatal care in the hospital). Enrolment into the study was done at the time of first antenatal visit. During enrolment, the study objective was described to the pregnant women and informed consents were obtained from them.As many as consented women were immediately enrolled into the study and given enrolment numbers with which they were monitored monthly for malaria parasite.

Thick and thin blood films were prepared for all blood samples and examined for malaria parasites by microscopy.

For the purpose of analysis, women who were positive for malaria parasites at one stage of pregnancy or the other were considered positive for malaria during pregnancy. Women positive for malaria in 1-3 months, 4-6months and 7-9 months were also considered positive in the first, second and third trimesters, respectively.

All data were entered into the computer and analysed using SPSS version 10.1 for windows. Descriptive statistics were computed for all relevant data. Associations were tested using Epi-Info 6.

\section{Results}

A total of 466 were enrolled in the study. Two hundred and ten $(45.1 \%)$ were from the traditional birth home (TBH) and two hundred and fifty-six (54.9\%) were from the two hospitals. A total of 140 primigravids were enrolled (72 from the hospital and 68 from the TBH). The multigravids totalled 325 (183 from the hospital and 142 from TBH) however; one of the women did not disclose her gravid status.

The age of the enrolled women in TBH ranged from 16-37years while in the hospital it was 1643years. The prevalence of malaria among the enrolled women at one stage of pregnancy or the other was $57.4 \%$; infection being higher among women attending the TBH (62.6\%) compared to those in the hospital $52.5 \%(P<0.05)$ (Table 1). A slightly higher

\footnotetext{
"To whom correspondence should be addressed. E-mail: etidowu@yahoo.com
} 
prevalence was recorded among primigravids (61.4\%) than multigravids $(55.6 \%)$. The difference was not significant $(P>0.05)$. The prevalence of malaria in the third trimester of pregnancy was higher $(47.5 \%)$ than for the first $(37.5 \%)$ and second $(47.3 \%)$ trimesters. However, there was no significant difference in the recorded the highest prevalence at booking than those in first and second trimesters, the difference was not significant. In the first trimester booking, infection was higher among hospital attendees while in the second and third trimester booking; prevalence was higher among TBH attendees.

Table 1: Malaria parasitaemia (\%) in different trimesters of pregnancy in Abeokuta

\begin{tabular}{lccccccccc}
\hline & \multicolumn{3}{c}{ Primigravidae } & \multicolumn{3}{c}{ Multigravidae } & \multicolumn{3}{c}{ Total } \\
& TBH & Hospitals & Total & TBH & Hospitals & Total & TBH & Hospitals & Total \\
\hline $1^{\text {st }}$ & 57.1 & 50.0 & 54.5 & 29.4 & 33.3 & 310 & 37.5 & 37.5 & 37.5 \\
$2^{\text {nd }}$ & 45.3 & 46.7 & 45.9 & 50.0 & 46.2 & 47.9 & 48.4 & 46.3 & 47.3 \\
$3^{\text {rd }}$ & 50.0 & 52.2 & 51.1 & 54.6 & 39.3 & 46.1 & 53.2 & 42.5 & 47.5 \\
Total & 63.2 & 55.6 & 61.4 & 62.8 & 63.4 & 55.6 & 62.9 & 52.5 & 57.4 \\
\hline
\end{tabular}

Table 2: Malaria parasitaemia (\%) at antenatal booking in Abeokuta

\begin{tabular}{lcccccccccccc}
\hline Parasiataemia & \multicolumn{3}{c}{ 1 $^{\text {st }}$ Trimester } & \multicolumn{3}{c}{ 2 $^{\text {nd }}$ Trimester } & \multicolumn{2}{c}{ 3 $^{\text {rd }}$ Trimester } & \multicolumn{2}{c}{ Total } \\
\hline Positive & 28.0 & 43.8 & 34.2 & 41.0 & 27.7 & 33.9 & 49.0 & 34.1 & 39.7 & 41.4 & 30.9 & 35.6 \\
Negative & 72.0 & 56.0 & 65.9 & 59.0 & 72.3 & 66.1 & 51.0 & 65.9 & 60.3 & 58.6 & 69.1 & 64.4 \\
Total (N) & 25 & 16 & 41 & 134 & 155 & 289 & 51 & 85 & 136 & 210 & 256 & 466 \\
\hline
\end{tabular}

Table 3: Parasitaemia (\%) at antenatal booking by gravid status in Abeokuta

\begin{tabular}{|c|c|c|c|c|c|c|c|c|c|c|c|c|}
\hline \multirow[t]{2}{*}{ Parasiataemia } & \multicolumn{3}{|c|}{$1^{\text {st }}$ Trimester } & \multicolumn{3}{|c|}{$2^{\text {nd }}$ Trimester } & \multicolumn{3}{|c|}{$3^{\text {rd }}$ Trimester } & \multicolumn{3}{|c|}{ Total } \\
\hline & Pri & $\begin{array}{l}\mathrm{Mu} \\
1\end{array}$ & Tot & Pri & Mul & Tot & Pri & Mul & Tot & Pri & $\begin{array}{c}\mathrm{Mu} \\
1\end{array}$ & Tot \\
\hline $\mathrm{MP}+\mathrm{ve}$ & 54.6 & 26.7 & 34.2 & 33.7 & 34.2 & 34.0 & 45.0 & 37.5 & 39.7 & 38.6 & 34.5 & 35.7 \\
\hline MP-ve & 45.4 & 73.3 & 65.8 & 66.3 & 65.8 & 66.0 & 55.0 & 62.5 & 60.3 & 61.4 & 65.5 & 64.3 \\
\hline Total & 11 & 30 & 41 & 89 & 199 & 288 & 40 & 96 & 136 & 140 & 34.5 & 465 \\
\hline
\end{tabular}

Key: Pri - primigravidae; Mul- multigravidae; Tot- total

prevalence of malaria in the three trimesters $(P>0.05)$. Infections in the second and third trimesters were slightly higher among women in the TBH and higher in the first and third trimesters among the primigravids $(P>0.05)$.

At the time of antenatal booking, $35.6 \%$ of the enrolled women were already parasitaemic with infection (Table 2). Although women who booked for antenatal care in the third trimester of pregnancy
The prevalence of infection among primigravids and multigravids was not significantly different at antenatal clinic booking $(P>0.05)$ (Table 3$)$.

The prevalence recorded at the ninth month of pregnancy $(35.7 \%)$ was not statistically different from that recorded at first antenatal visit $(P>0.05)$. There was however a decrease in malaria prevalence among primigravids and an increase among multigravids between the first ANC visit and the ninth month of pregnancy (Table 4).

Table 4: Prevalence of malaria infection at first ANC visit and the ninth month of pregnancy at Abeokuta

\begin{tabular}{|c|c|c|c|c|c|c|c|c|c|}
\hline & \multicolumn{3}{|c|}{ Traditional Birth Home } & \multicolumn{3}{|c|}{ Hospital } & \multicolumn{3}{|c|}{ Total } \\
\hline & $\begin{array}{l}\text { Primi } \\
(\%)\end{array}$ & $\begin{array}{l}\text { Mult } \\
(\%)\end{array}$ & $\begin{array}{l}\text { Total } \\
(\%)\end{array}$ & $\begin{array}{l}\text { Primi } \\
(\%)\end{array}$ & $\begin{array}{l}\text { Mult } \\
(\%)\end{array}$ & $\begin{array}{l}\text { Total } \\
(\%)\end{array}$ & $\begin{array}{l}\text { Primi } \\
(\%)\end{array}$ & $\begin{array}{l}\text { Mult } \\
(\%)\end{array}$ & $\begin{array}{c}\text { Total } \\
(\%)\end{array}$ \\
\hline $\begin{array}{l}\text { At 1st ANC } \\
\text { Visit }\end{array}$ & 50.0 & 30.3 & 36.2 & 40.0 & 32.4 & 35.1 & 44.1 & 31.4 & 35.6 \\
\hline At $9^{\text {th }}$ month & 39.7 & 41.7 & 41.4 & 37.5 & 28.4 & 30.9 & 38.6 & 34.5 & 35.7 \\
\hline
\end{tabular}

Key: Primi= primigravidae; Multi= multigravidae 


\section{Discussion}

The prevalence of malaria $(57.4 \%)$ recorded in this study is an indication that malaria in pregnancy is still a serious problem in Abeokuta. Pregnancy has been identified to increase the risk and vulnerability to malaria infection. The prevalence of malaria among pregnant women recorded in this study is higher than the ones recorded by Matteelli et al. (1994) and Akanbi et al. (2004) in cross-sectional studies carried out in Ibadan, Nigeria. The higher prevalence recorded in this study may be attributed to the inclusion of women receiving antenatal care in the $\mathrm{TBH}$ which were observed to have a higher prevalence compared to women in the hospital. Moreover, this was a longitudinal study and included a larger sample. In a cross-sectional study, women who were not parasitaemic at one stage of pregnancy may likely to be parasitaemic in subsequent stages, contributing to lower parasitaemia rates observed in the Ibadan studies.

The higher prevalence recorded among primigravids than multigravids conforms to findings by other workers in Nigeria (Archibald, 1956). The author reported higher malaria prevalence among primigravids than multigravids. This higher level of susceptibility in primigravids has been reported to be common in highly endemic areas (Garner \& Gulmezoglu, 2001). Although the difference in prevalence between the gravid types recorded in our study was not statistically significant, this may indicate that there may be susceptibility in multgravids as well as in primigravids observed in a study by Shulman et. al. (2001).

Various workers have reported high prevalence of malaria in different trimesters of pregnancy. Brabin (1983) reported higher infection in the second trimester of pregnancy whereas D'Allesandro \& Langerock (1998) in Mali identified risk of malaria infection to be significantly higher in the first trimester of gestation. In our study however, we recorded the least prevalence in the first trimester of pregnancy and the highest in the third trimester. The reason for this variation could not be determined but is likely to be linked to the small sample size obtained in the first trimester since most women came for antenatal care in the second trimester. The fact that the difference in malaria parasitaemia between different stages of pregnancy recorded in this study is not statistically significant implies that women are at equal risk of malaria infection.

Malaria infection was observed to be higher among women in the TBH even in different trimesters of pregnancy. The higher prevalence in the TBH could be due to the type of management given to them, which was mainly in the use of herbal preparation of various types of undisclosed plants; these preparations may not be sufficient to give the necessary malaria prevention and/or treatment.

In this study, the highest malaria prevalence at booking was recorded among women who booked for ANC in the third trimester indicating that late ANC booking may predispose more to malaria infection during pregnancy. This behaviour does not provide early prevention and management of malaria in form of malaria prophylactic drugs given especially in the hospitals. Primigravids that book late for ANC may be at a higher risk of malaria during pregnancy since infection at booking was even higher among primigravids. There seem to be no positive impact of antenatal care on malaria prevalence as there was no significant difference in the prevalence. This may have been influenced by the late $\mathrm{ANC}$ registration and poor attendance. The need to improve the quality of care, malaria prevention and treatment given to pregnant women especially those receiving antenatal care outside the hospital is recommended. Women should also be encouraged to book early for antenatal.

\section{References}

Akanbi, O.M., Odaibo, A.B. Afolabi, K. \& Ademowo, O.G. (2004) Prevalence of malaria and anaemia in pregnancy in Ibadan South Western Nigeria. Nigerian Journal of Parasitology 25, 51-55.

Archibald, H.M. (1956) The influence of malaria infection of the placenta on the incidence of prematurity. Bulletin of World Health Organization 15, 842-845.

Brabin, B.J. (1983) An analysis of malaria in pregnancy in Africa. Bulletin of the World Health Organization 61, 1005 - 1016.

D’ Alessandro, U. \& Langerock, P. (1998) The Impact of a national impregnated bednet programme on the outcome of pregnancy in primigravidae in The Gambia. Transactions of the Royal Society of Tropical Medicine and Hygiene $\mathbf{9 0}$, 487-492.

Ebrahim, G.J. (1996) Malaria during pregnancy. Journal of Tropical Paediatrics 42, 62-63.

Garner, P. \& Gulmezoglu, A. (2001) Prevention versus treatment for malaria in pregnant women. In the Cochrane Library Oxford; Update Software 3. 
Leontsini, E. (1994) Women and Malaria. PVO Child Survival Technical Report 4 (1): 7-8.

Mattelli, A., Onsto, F. \& Shein, A. (1994) Malaria and anaemia in pregnant women in Urban Zanzibar, Tanzania. Annals of Tropical Medicine and Parasitology 88, 475- 483.

Shulman, C.E., Marshol, T., Dorman, E.K., Bulmer, J.N., Cutts, F., Peshu, N. \& Marsh, K. (2001) Malaria in pregnancy: adverse effects on haemoglobin levels and birthweight in primigravidae. Tropical Medicine and International Health 6, 770-778.
Steketee, R., Wirima, J. J. \& Campbell, C. (1996) Developing effective strategies for malaria prevention programs for pregnant African Women. American Journal of Tropical Medicine and Hygiene 55, 95-100.

Taha, T.E. \& Gray, R.H. (1993) Malaria and perinatal mortality in Central Sudan. American Journal of Epidemiology 15, 563-568.

UNICEF (2000) Roll Back Malaria A Global Partnership. Rolling Back Malaria. United National International Children Fund (UNICEP). 17 pp.

WHO (1998) Malaria Know the Facts. World Health Organization Newsletter 13 (1) 6-7. 